\title{
Response variability patterns in complex tasks
}

\author{
LOWELL T. CROW, DAVE A. LOWIN, L. ROBERT VAN AUSDLE, and KRIS M. WALTON \\ Western Washington University, Bellingham, Washington
}

\begin{abstract}
Fifty-three subjects were given a video block-design task, and measures were made of beat-tobeat latencies of heart rate both during the task and during a subsequent rest period. The results indicated a decreased heart rate variance in the puzzle-solving period when compared to heart rate in the resting period. In addition, relationships were found between heart rate and other response variances for males. The results are discussed in terms of a behavioral variability hypothesis of mild stress effects (Crow, 1977).
\end{abstract}

Rats subjected to aversively conditioned stimuli show a diminished uncertainty in operant responding (Crow, Bendt, \& Tracy, 1982), and moderate doses of ethanol have been shown to reduce both rats' uncertainty of responding (Crow, McWilliams, \& Ley, 1979) and performance in a radial-arm maze (Devenport \& Merriman, 1983). As is the case with the rat, it has been found that alcohol diminishes man's uncertainty of responding with a paddle controller in a videogame task (Crow \& Hirdler, 1985).

These findings are consistent with an earlier proposal by Crow (1977) that alcohol and aversive stimuli both may constitute mild stress or excitement and, thus, may provide the basis for response-variability reduction. The present study was designed to examine the effects on human response variability of other situations that might be considered mildly stressful. A difficult video block-design puzzle was chosen, and measures were made of heart rate as the primary response variable.

\section{METHOD}

\section{Subjects}

Thirty-three female and 20 male university students participated.

\begin{abstract}
Apparatus
The variance of the latency between heartbeats was obtained using a Grass PTT1 Photocell Transducer, 7 P9A Photocell Transducer Matching Panel, and an Atari PC.

\section{Procedure}

Subjects were given a self-report questionnaire that required about 5 min to complete, and then were given instructions on a video blockdesign puzzle. Four different puzzles were presented, each with a 3min time limit during which the subject's heart rate and uncertainty of movements of a joystick were measured. At the conclusion of the puzzlesolving period, which was approximately $15 \mathrm{~min}$, a resting heart rate record was obtained for an additional $5 \mathrm{~min}$. The session for each subject was approximately $30 \mathrm{~min}$ in duration.
\end{abstract}

The paper was originally presented at the annual meetings of the $\mathrm{Pa}$ cific Cascade Chapter of the Society for Neuroscience, Sublimity, OR, May, 1986. The authors' mailing address is: Department of Psychology, Western Washington University, Bellingham, WA 98225.

\section{RESULTS}

The heart rate data were obtained by collecting means and variance of beat-to-beat latencies in seconds. These means and variances were computed on-line for each 1-min segment of the puzzle task and the rest period. The mean heart rate variances for these two conditions are shown in Figure 1. These differences are significant for both men $[t(19)=2.96, p<.03]$ and women $[t(32)=$ $3.34, p<.01]$. The mean heart rate also was significantly different during the rest period and puzzle task (lower in the rest period than in the puzzle-solving period) for both sexes.

The self-rating form consisted of 11-point scales for each month for the past 3 years. The subject was instructed to consider whether a particular month represented an "up" or a "down" in his/her life and to circle the number that indicated the degree of his/her feelings. The variance of these ratings (FORMV) was obtained by use of all ratings for each subject.

Uncertainty scores (Crow, McWilliams, \& Ley, 1979) were computed for each block-design test by assessing the predictability of sequences of movements of the

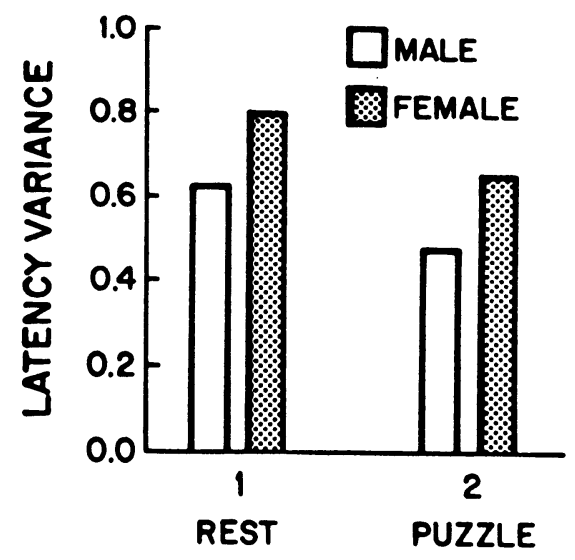

\section{CONDITIONS}

Figure 1. Heart rate variance scores in the resting and puzzle task conditions for males and females. 
Table 1

Correlation Coefficients of Each Variance Measure with Every Other

\begin{tabular}{lcc} 
& Males & Females \\
\hline FORMV \& PUZU & .055 & .008 \\
PUZHRV \& RESTHRV & .732 & .856 \\
PUZHRV \& PUZU & .149 & -.117 \\
FORMV \& PUZHRV & .513 & .000 \\
FORMV \& RESTHRV & .701 & .027 \\
PUZU \& RESTHRV & .107 & -.081 \\
\hline
\end{tabular}

Note-FORMV = self-rating form variance; PUZU = mean uncertainty scores for movements of the joystick in the block-design task; PUZHRV = mean heart rate variance during the block design; RESTHRV = mean heart rate variance during the rest period.

joystick itself and the joystick button. These U-scores (PUZU) were computed on-line for each 3-min game period.

Correlation coefficients of each variability measure with every other variability measure were calculated for both males and females, and the results are shown in Table 1. It can be seen that for a particular individual, response variabilities are predictive of other response variabilities only in the case of self-ratings and heart rate and, for those, only in men.

\section{DISCUSSION}

An underlying assumption of the response-variability hypothesis (Crow, 1977) is that impositions upon particular responses will result in a reduction in the kinds of activities constituting that behavioral class and, thus, result in a reduction of response variability. A "mapping" of such response-variability-reducing impositions includes drug effects, reinforcement, and certain kinds of aversive stimulation. It is thought that stress, in the sense used by Hans Selye, may underlie all these conditions and that perhaps one might add to Selye's stress effects those of response stereotypy.
An earlier attempt (Crow \& Hirdler, 1985) to show relationships between the Spielberger Anxiety State-Trait Questionnaire and objective measures of response variability failed, but the Anxiety State-Trait Questionnaire yields no response-variability data. For the present study, a "stress questionnaire" was devised that would yield both means and variability data.

It is interesting that such questionnaire variability data do, in some cases, correlate with the heart rate variability, whereas the mean questionnaire scores (not reported here) do not. The sex differences in these correlations are striking, and one can only speculate as to their basis. It is possible that women react in a less stressful manner to the questionnaire, which concerns self-ratings of month-to-month "ups and downs," than do men. Given this and the apparent fact that both considered the puzzle equally stressful (fewer than $1 \%$ of the subjects were successful in solving one of the four problems), the higher correlations for men would be expected.

Unlike the videogame task studied earlier using a paddle controller (Crow \& Hirdler, 1985), the uncertainty scores computed from the joystick used in the puzzle of the present study yielded very little betweensubjects variance, and thus did not discriminate between subjects. The variability patterns for this task were not derivative of accuracy of performance, as was the case in the earlier study. That is, the ideal problemsolving sequence of responding was not a first-order stereotypic one but necessitated a certain degree of variance from a set pattern. It is possible that such task-demanded uncertainty data do not reflect general behavioral variability, but rather variability specific to that activity.

\section{REFERENCES}

Crow, L. T. (1977). Is variability a unifying behavioral concept? The Psychological Record, 27, 783-790.

Crow, L. T., BendT, V. A. H., \& Tracy, D. M. (1982). Mutually antagonistic effects of ethanol and an aversive CS+. Bulletin of the Psychonomic Society, 20, 263-265.

Crow, L. T., \& HiRdLer, K. R. (1985). Alcohol effects on the variability of performance in a videogame task. Bulletin of the Psychonomic Society, 23, 519-520.

Crow, L. T., McWilliams, L. S., \& Ley, M. (1979). Relative stereotypy of water-ingestive behavior induced by chronic alcohol injections in the rat. Bulletin of the Psychonomic Society, 14, 278-280.

DEVENPORT, L. D., \& MERRIMAN, V. J. (1983). Ethanol and behavioral variability in the radial-arm maze. Psychopharmacology, 79, 21-24. 